\title{
Editorial
}

Ophthalmologe 2020 $117: 843-845$

https://doi.org/10.1007/s00347-020-01206-2

(c) Springer Medizin Verlag GmbH, ein Teil von Springer Nature 2020

Liebe Leserinnen und Leser, liebe Mitglieder der DOG,

es freut mich, Ihnen mitzuteilen, dass das Gesamtpräsidium der Deutschen Ophthalmologischen Gesellschaft in diesem Jahr 2 außergewöhnliche Persönlichkeiten der Augenheilkunde für ihr großes Engagement für unser Fach und für unsere Fachgesellschaft durch die Verleihung der Ehrenmitgliedschaft gewürdigt hat. $\mathrm{Da}$ in diesem Jahr die Verleihung nicht im gewohnten Rahmen unseres Jahreskongresses stattfinden kann, möchte ich Ihnen auf diesem Wege die beiden neuen Ehrenmitglieder vorstellen, die sicherlich bereits vielen von Ihnen bekannt sind. Beide haben sich - jeder auf seinem Gebiet - besonders für die Augenheilkunde eingesetzt, und es ist mir eine große Freude, dass ich dieses Jahr die Ehrenmitgliedschaft der DOG an Prof. Dr. Volker Klauß und Prof. Dr. Jens Martin Rohrbach verleihen darf.

Prof. Dr. med. Volker Klauß (• Abb. 1) arbeitete bis 2007 als Professor an der Universitätsaugenklinik der Ludwig-Maximilians-Universität in München.

\section{Hans Hoerauf}

Augenklinik, Universitätsmedizin Göttingen, Göttingen, Deutschland
Nach Studium, Promotion und Approbation in Göttingen, München und Paris war Prof. Klauß über den Deutschen Entwicklungsdienst von 1970 bis 1972 als Distriktarzt am Krankenhaus Mbarara in Uganda, Afrika, als Allgemeinarzt und Geburtshelfer tätig, in einem damals sehr unsicheren Umfeld kurz nach dem Putsch. Sein Engagement für Menschen mit Augenerkrankungen in Afrika zieht sich durch sein gesamtes Berufsleben. Die Facharztanerkennung als Ophthalmologe erlangte er 1976 in München. Ein Lehrauftrag führte ihn 1978 schließlich wieder nach Afrika zurück. An der Universität Nairobi gründete Prof. Klauß mit Unterstützung durch Prof. O.E. Lund die Kooperation der Medizinischen Fakultät der LMU und der Universität Nairobi, was in die Gründung einer Abteilung für Augenheilkunde an der Universität von Nairobi mündete. Dort bildete er von 1978 bis 1985 als Lecturer und Senior-Lecturer zahlreiche afrikanische $\mathrm{Au}$ genärzte aus. Über 230 Augenärzte aus 14 afrikanischen Ländern sind in diesem langfristigen, von dem DAAD geförderten Entwicklungsprojekt inzwischen ausgebildet worden.
$\mathrm{Zu}$ seinen wichtigsten Forschungsgebieten zählen die präventive Ophthalmologie und die Tropenophthalmologie.

Fast 2 Jahrzehnte lang war Professor Klauß Vorsitzender des Deutschen Komitees zur Verhinderung von Blindheit. Er unterstützte die europäische Sektion der International Agency for the Prevention of Blindness als Chairman, die WHO als Berater und ist Mitglied des Beirats des International Council of Ophthalmology (ICO). Bis heute engagiert sich Prof. Klauß im ärztlichen Aus- und Weiterbildungsprogramm der Universität Nairobi sowie in weiteren afrikanischen Ländern wie Äthiopien, Uganda, Malawi, Kamerun und in den letzten Jahren auch in Asien, v. a. in Myanmar.

Auch als Wissenschaftler setzte sich Prof. Volker Klauß im Kampf für den Erhalt des Augenlichts und gegen Erblindung in Ländern der Dritten Welt ein. Im Jahr 2008 erhielt Volker Klauß für sein Wirken das Bundesverdienstkreuz am Bande.

Innerhalb der DOG engagierte sich Prof. Klaußin der Arbeitsgruppe Internationale Ophthalmologie (gegründet 1994

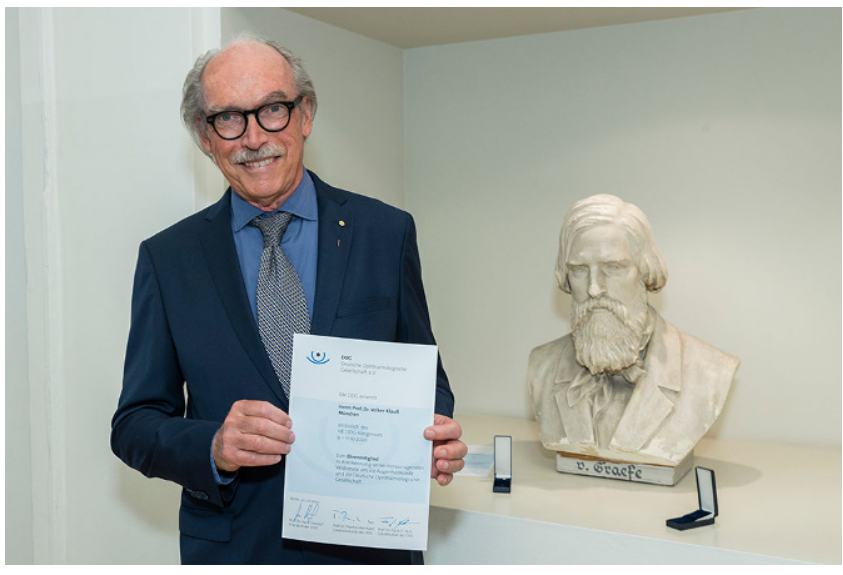

Abb. 1 ॥ Prof. Dr. med. Volker Klauß. (@ DOG/Deckbar)

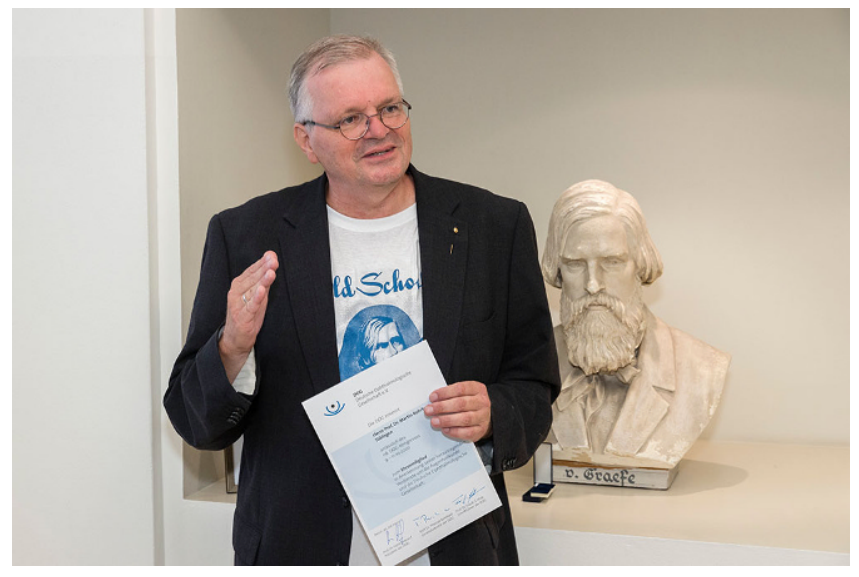

Abb. 2 ム Prof. Dr. med. Jens Martin Rohrbach. (@ DOG/Deckbar) 


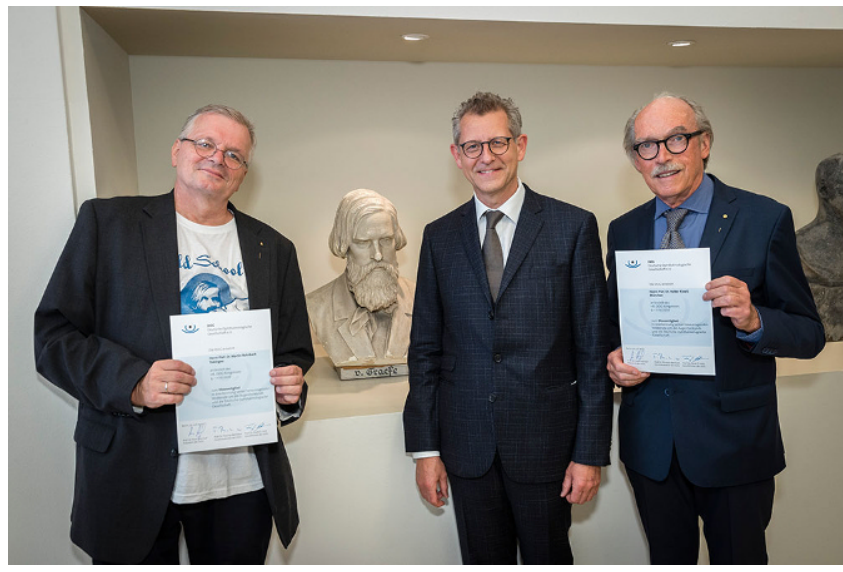

Abb. $3<$ V.I.n.r.: Prof. Rohrbach, Prof. Hoerauf, Prof. Klauß. (৫ DOG/Deckbar)

in Mannheim) und seit 2004 in der Sektion Internationale Ophthalmologie.

Prof. Volker Klauß hat sich über Jahrzehnte für die Augenheilkunde in Entwicklungsländern eingesetzt und eine enge Zusammenarbeit aufgebaut. Die Deutsche Ophthalmologische Gesellschaft ist ihm hierfür zu großem Dank verpflichtet.

Prof. Dr. med. Jens Martin Rohrbach (- Abb. 2) hat von 1974 bis 1980 an der Eberhard-Karls-Universität in Tübingen Humanmedizin studiert. Wesentliche Station vor seinem Eintritt in die klinische Weiterbildung in der Augenheilkunde waren 2 aktive wissenschaftlich geprägte Jahre am Anatomischen Institut der Friedrich-Alexander-Universität in Erlangen. Unter der Leitung von Herrn Prof. Dr. J.W. Rohen und der Betreuung durch Frau Prof. Dr. Lütjen-Drecoll beschäftigte er sich insbesondere mit der Morphologie des Kammerwinkels, aber auch mit der Embryologie des Auges. Im Jahr 1984 kehrte er zurück an die Univ.Augenklinik nach Tübingen für seine Weiterbildung im Fach Augenheilkunde unter der Leitung von Herrn Prof. H.J. Thiel. In Tübingen war er von Beginn an mit der Ophthalmopathologie befasst. Nach Anerkennung als Facharzt im Jahr 1988 und dem Beginn der oberärztlichen Tätigkeit ab 1989 habilitierte er im Februar 1993 im Fach Augenheilkunde an der Medizinischen Fakultät der Eberhardt Karls Universität Tübingen mit dem Thema „Morphologische Studien zum Wachstum des malignen Aderhautmelanoms“. Im Jahr 1994 übernahm er die Leitung des von Prof. Stock gegründeten und Prof. Naumann intensiv weiter ausgebauten ophthalmopathologischen
Labors der Tübinger Augenklinik. Seit 1999 ist er „außerplanmäßiger Professor" der Eberhardt-Karls-Universität. Im Jahr 2000 vertrat er kommissarisch den Ärztlichen Direktor der UniversitätsAugenklinik in Kiel. Forschungsschwerpunkte von Herrn Prof. Rohrbach liegen v. a. in der Ophthalmopathologie von Erkrankungen der Hornhaut, Tumoren des Auges und dem Sekundärglaukom. Er ist Autor von über 230 Originalarbeiten und mehreren Lehrbüchern. Als Sprecher der Sektion Ophthalmopathologie der DOG hat er engagiert die Arbeiten des Gesamtpräsidiums der DOG unterstützt.

Prof. Jens Martin Rohrbach hat sich jahrzehntelang der Geschichte der Augenheilkunde gewidmet und deren $\mathrm{Ge}$ genwart und Zukunft stets im historischen Kontext reflektiert. Besonders hervorzuheben ist sein Engagement für die Aufarbeitung der Geschichte der Augenheilkunde im Nationalsozialismus. Seine Monographie von 2007 „Augenheilkunde im Nationalsozialismus" fand große Beachtung. Jens Martin Rohrbachs Aufarbeitung der NS-Geschichte fand auch in der Festschrift von 2007 „150 Jahre DOG“ Niederschlag.

Mit großem Engagement bearbeitete Jens Martin Rohrbach historische Grundlagen und Zusammenhänge des heutigen Faches Augenheilkunde und seiner Fachgesellschaft. Unvergessen bleibt seine Festrede „Danken - Gedenken - Gedanken“ aus dem Jahr 2007 anlässlich des 150. Geburtstages der DOG (105. Tagung) zur Geschichte unserer Fachgesellschaft.
Im Jahr 2013 gab Prof. Rohrbach den Schriftwechsel Graefe - Donders (1852-1870) zusammen mit dem Historiker Thomas Schilp heraus. Das Buch beginnt mit der Biografie Albrecht von Graefes, die Prof. Rohrbach nach intensiver und aufwendiger Analyse historischer Originalliteratur verfasste. Ebenso wie die Aufarbeitung der NS-Zeit stellt dieses Buch zum Briefwechsel Graefe - Donders für die DOG einen wichtigen Meilenstein augenheilkundlicher Geschichte dar.

Im Jahr 2016 wurde Prof. Jens Martin Rohrbach der „Von-Graefe-Preis“ für ganz besonders herausragende wissenschaftliche Leistungen im Bereich der Augenheilkunde im deutschsprachigen Raum zuerkannt, und als Preisträger hielt er im Folgejahr während der Jahrestagung der DOG die Von-Graefe-Vorlesung.

Zum 150. Todestag Albrecht von Graefes hat Prof. Jens Martin Rohrbach sein umfassendes Wissen in eine aktuelle und sehr lesenswerte Biografie einfließen lassen. Es ist ihm gelungen, den Menschen von Graefe aus verschiedenen Blickwinkeln zu beleuchten und nicht nur ein berufliches, sondern auch ein privates Bild von Graefes vor dem geistigen Auge des Lesers entstehen zu lassen.

Die Deutsche Ophthalmologische Gesellschaft ist Prof. Rohrbach zu großem Dank verpflichtet, und es ist mir eine besondere Freude, ihn gerade im Graefe-Jahr mit der Ehrenmitgliedschaft der DOG zu würdigen.

Prof. Dr. med. Volker Klauß und Prof. Dr. med. Jens Martin Rohrbach haben sich über Jahrzehnte für die Deutsche Ophthalmologische Gesellschaft eingesetzt und große Verdienste erworben. Es ist mir eine besondere Freude, ihnen im Graefe-Jahr 2020 die Ehrenmitgliedschaft der Deutschen Ophthalmologischen Gesellschaft zu verleihen (• Abb. 3 ).

Ihr

Hans Hoerauf

Präsident der Deutschen Ophthalmologischen Gesellschaft 


\section{Korrespondenzadresse}

\section{Prof. Dr. med. Hans Hoerauf, FEBO}

Augenklinik, Universitätsmedizin Göttingen Robert-Koch-Str. 40, 37075 Göttingen, Deutschland

Hans.Hoerauf@med.uni-goettingen.de

Interessenkonflikt. H. Hoerauf gibt an, dass kein Interessenkonflikt besteht.

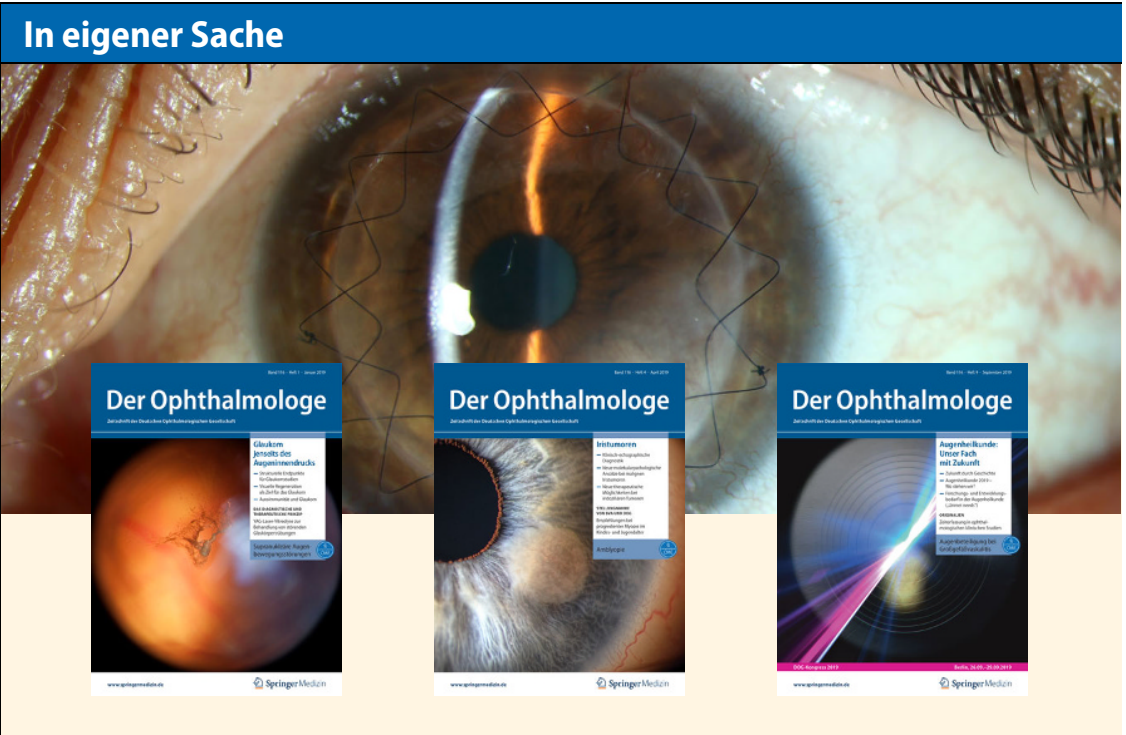

Leitthemenübersicht von Der Ophthalmologe

Der Ophthalmologe bietet Ihnen jeden Monat umfassende und aktuelle Beiträge zu interessanten Themenschwerpunkten aus allen Bereichen der Augenheilkunde.

Rückblick - 2019

01/19 Glaukom jenseits des

Augeninnendrucks

02/19 Neurotrophe Keratopathie

03/19 Therapie der Fuchs-Endotheldystrophie mittels DMEK

04/19 Iristumoren

05/19 Komplexe Glaukome mit hohem Behandlungsrisiko

06/19 Myopie - Epidemiologie,

Pathomechanismen und Prävention

07/19 Originalien

08/19 OCT-Angiographie und systemische Erkrankungen

09/19 Augenheilkunde: unser Fach mit Zukunft

10/19 Schwere Flüssigkeiten in der Netzhautchirurgie

11/19 Makulaformen und andere Makulopathien

12/19 Augenverletzungen durch Feuerwerks- und Knallkörper

SpringerMedizin.de bietet Ihnen Zugang zu allen elektronisch verfügbaren Ausgaben Ihrer Zeitschrift - unabhängig davon, seit wann Sie die Zeitschrift abonniert haben.

\section{Rückblick \& Vorschau - 2020}

01/20 Originalien

02/20 Periokuläres Basalzellkarzinom

03/20 Maximalinvasive Vorderabschnittschirurgie im Kindesalter 04/20 Qualitätssicherung bei der IVOM 05/20 Der Femtosekundenlaser in der Kataraktchirurgie

06/20 Personalisierte Medizin in der Behandlung von Augentumoren 07/20 SARS-CoV-2-Infektion und Auge 08/20 Space-Eye-Disease: Weltall, Auge und Glaukom

09/20 Retinologische Notfälle - das richtige Timing

10/20 Künstliche Intelligenz in der Augenheilkunde

11/20 Vergleichende Gegenüberstellung von Seh- und Hörbahn

12/20 Mikrochirurgische Operationstechniken aus ophthalmopathologischer Sicht

(Änderungen vorbehalten)

Alle Inhalte der Zeitschrift finden Sie unter www.springermedizin.de/ der-ophthalmologe 